\title{
Aarroo Perfil dos agravos relacionados ao trabalho notificados no Rio Grande do Norte, 2007 a 2009*
}

doi: $10.5123 /$ S1679-49742014000400016

\section{Profile of work-related injuries reported in the state of Rio Grande do Norte, Brazil, 2007-2009}

Cleonice Andréa Alves Cavalcante

Escola de Enfermagem de Natal, Universidade Federal do Rio Grande do Norte, Natal-RN, Brasil

Renata Silva Santos

Departamento de Enfermagem, Universidade Federal do Rio Grande do Norte, Natal-RN, Brasil

Elisângela Franco de Oliveira Cavalcante

Escola de Enfermagem de Natal, Universidade Federal do Rio Grande do Norte, Natal-RN, Brasil

Rogéria Lima Martins

Vigilância Epidemiológica, Secretaria de Estado da Saúde Pública do Rio Grande do Norte, Natal-RN, Brasil

Erika Aparecida da Silveira

Faculdade de Medicina, Universidade Federal de Goiás, Goiânia-GO, Brasil

Erica Tatiane da Silva

Secretaria de Vigilância em Saúde, Ministério da Saúde, Brasília-DF, Brasil

\section{Resumo}

Objetivo: descrever o perfil dos agravos relacionados ao trabalho notificados no estado do Rio Grande do Norte, Brasil, de 2007 a 2009. Métodos: estudo descritivo, com dados do Sistema de Informação de Agravos de Notificação do Centro de Referência em Saúde do Trabalhador. Resultados: dos 167 municípios, 40,1\% tiveram notificações de doenças e agravos, entre acidentes biológicos $(53,7 \%)$, acidentes graves $(38,4 \%)$, distúrbios oesteomusculares $(3,1 \%)$, intoxicações exógenas $(2,6 \%)$, transtornos mentais $(1,5 \%)$, pneumoconioses $(0,5 \%)$ e dermatoses $(0,2 \%)$; entre acidentados, predominaram trabalhadores do sexo masculino (53,7\%), de 25 a 44 anos de idade (62,1\%), escolaridade até o Ensino Médio (25,9\%), com emprego registrado $(23,9 \%)$; incapacidades temporárias corresponderam a 32,0\% dos registros. Conclusão: evidenciou-se o perfil e o impacto das doenças e agravos ocupacionais no estado e a importância de dados consistentes e oportunos para seu monitoramento sistemático e o planejamento das ações em Saúde do Trabalhador.

Palavras-chave: Epidemiologia Descritiva; Doenças Profissionais; Acidentes de Trabalho; Saúde do Trabalhador.

\section{Abstract}

Objective: to describe the epidemiological profile of notified work-related health problems in Rio Grande do Norte State, Brazil, 2007-2009. Methods: this was a descriptive study using Occupational Health Reference Center data kept on the Notifiable Diseases Information System. Results: 40.1\% of the 167 municipalities notified occupational diseases, as follows: biological accidents (53.7\%), serious accidents (38.4\%), Musculoskeletal Disorders (3.1\%), exogenous poisoning (2.6\%), mental disorders (1.5\%), pneumoconiosis (0.5\%) and dermatoses (0.2\%). The most affected were male workers (53.7\%), people aged 25-44 (62.1\%), those with middle school education (25.9\%) and those having formal employment (23.9\%). 32.0\% of cases reported temporary incapacity. Conclusion: the profile and impact of occupational injuries and diseases in the State were revealed, as was the importance of consistent and timely data for their systematic monitoring and planning of Occupational Health actions.

Key words: Descriptive Epidemiology; Occupational Diseases; Occupational Accidents; Occupational Health.

* Artigo desenvolvido como pré-requisito para conclusão do Curso de Especialização em Epidemiologia, por Cavalcante CAA, Santos RS, Cavalcante EFO e Martins RL, na modalidade de Ensino a Distância - Polo Natal -, financiado com recursos da Secretaria de Vigilância em Saúde do Ministério da Saúde e executado pela Universidade Federal de Goiás.

Endereço para correspondência:

Cleonice Andréa Alves Cavalcante - Av. Gandhi, n 1652, Residencial Santa Sofia, Casa 13, Nova Parnamirim, Parnamirim-RN Brasil. CEP: 59152-780

E-mail:cleoandreaeen@gmail.com 


\section{Introdução}

A exposição dos trabalhadores a riscos físicos, químicos, ergonômicos, psicossociais e/ou biológicos advindos da execução do trabalho pode resultar em doenças e agravos ocupacionais. Apesar de representar uma dimensão fundamental na estruturação do homem e da sociedade, o trabalho, tanto no que se refere ao desenvolvimento de suas capacidades como à garantia das condições materiais de sobrevivência, tem sido, ao longo dos tempos, provocador de sofrimentos, adoecimentos e morte. ${ }^{1,2}$

Os trabalhadores adoecem e morrem por causas relacionadas ao trabalho, como consequência direta das atividades profissionais que exercem ou exerceram, ou pelas condições adversas em que seu trabalho é ou foi realizado. Dessa forma, o trabalho impacta sobre o perfil de morbimortalidade dos trabalhadores, contribuindo, de forma direta - os acidentes de trabatho e as chamadas doenças profissionais - e indireta, nas chamadas doenças relacionadas com 0 trabalho. ${ }^{2}$

\section{A exposição dos trabalhadores ariscos físicos, químicos, ergonômicos, psicossociais e/oubiológicos advindos da execução dotrabalho pode resultar em doençase agravos ocupacionais.}

Em 2013, a Organização Internacional do Trabalho (OIT) estimou que anualmente, 160 milhões de trabalhadores são atingidos por doenças ocupacionais no mundo e 2 milhões morrem de doenças e/ou acidentes ocorridos no ambiente de trabalho. Portanto, mais de 5 mil pessoas morrem a cada dia por problemas relacionados ao trabalho. A OIT também estimou que os acidentes de trabalho e as doenças profissionais resultam em uma perda anual de $4 \%$ no produto interno bruto (PIB) mundial, ou cerca de 2,8 bilhões de dólares, em custos diretos e indiretos relacionados a lesões e doenças. Nesse contexto, o Brasil ocupa 0 quarto lugar no mundo quanto ao risco de morte no trabalho. ${ }^{3}$

No Brasil, a despeito da ausência de informações precisas, os agravos relacionados ao trabalho constituem graves problemas de Saúde Pública. A escassez e a inconsistência das informações sobre a real situação de saúde dos trabalhadores dificultam a definição de prioridades para as políticas públicas, o planejamento e a implementação das ações de Saúde do Trabalhador, além de privar a sociedade de subsídios importantes para a melhoria das condições de vida e trabalho. No caso do sistema de informação em saúde, ressalta-se o evidente sub-registro de agravos relacionados ao trabalho, uma vez que seu número é muito baixo quando comparado ao de países desenvolvidos. ${ }^{4}$

0 estudo desses agravos relacionados ao trabalho tem se mostrado de importância fundamental no cenário das atividades de vigilância em saúde, pois permite a realização do diagnóstico da ocorrência do evento na população, fornecendo subsídios para explicações causais dos agravos de notificação compulsória, além de indicar os riscos aos quais as pessoas estão sujeitas, contribuindo para a identificação da realidade epidemiológica de determinada área geográfica. Trata-se de um instrumento relevante para auxiliar o planejamento da saúde, definir prioridades de intervenção, além de permitir que seja avaliado o impacto das intervenções.

A disponibilização de informações sobre o perfil dos trabalhadores e a ocorrência dos agravos relacionados ao trabalho tem o potencial de subsidiar ações no campo da Saúde do Trabalhador, especialmente no planejamento e organização dos serviços e na proposição de estratégias de educação e prevenção. 0 presente estudo tem como objetivo descrever o perfil epidemiológico dos agravos relacionados ao trabalho notificados no Sistema de Informação de Agravos de Notificação (Sinan) do estado do Rio Grande do Norte, no período de 2007 a 2009.

\section{Métodos}

Estudo descritivo desenvolvido junto ao Centro de Referência em Saúde do Trabalhador (Cerest) da Vigilância Epidemiológica da Secretaria de Estado da Saúde Pública do Rio Grande do Norte.

0 estado do Rio Grande do Norte está situado na região Nordeste, tem 167 municípios distribuídos em $52.811 \mathrm{~km}^{2}$, o que equivale a 3,42\% da área do Nordeste e a $0,62 \%$ da superfície do Brasil, e uma população estimada de 3.373.959 de habitantes. Oficialmente, a única região metropolitana do estado é a Região Metropolitana de Natal, cujos municípios integrantes são Natal, Parnamirim, São Gonçalo do Amarante, Ceará-Mirim, Macaíba, Extremoz, São José de Mipibu, Nísia Floresta, Monte Alegre e Vera Cruz. 
A Rede Nacional de Atenção Integral à Saúde do Trabalhador (Renast) é composta pelos (i) Centros Estaduais e Regionais de Referência em Saúde do Trabalhador - Cerest -, polos de suporte técnico-científico no processo de trabalho/saúde/doença que desenvolvem ações de prevenção e vigilância pela melhoria das condições de trabalho e da qualidade de vida dos trabalhadores, e por (ii) redes sentinelas de serviços médicos e ambulatoriais de média e alta complexidade, responsáveis por diagnosticar acidentes e doenças relacionados ao trabalho e registrá-los no Sinan-net, sistema desenvolvido para a coleta e divulgação dos dados gerados pela rotina da vigilância epidemiológica.

0 processo de autorização e liberação dos dados pelo Cerest estadual ocorreu durante o mês de setembro de 2010, incluindo os dados lançados até a $37^{\mathrm{a}}$ semana epidemiológica, realizada no dia $18 \mathrm{de}$ setembro de 2010. Considerando-se que o registro no Sinan dos agravos relacionados ao trabalho de investigação, conforme preconizado pela Portaria do Ministério da Saúde (GM/MS) $\mathrm{n}^{0} 777$, de 28 de abril de 2004, ${ }^{5}$ foi implementado no Rio Grande do Norte a partir de 2007, este foi o ano do início do levantamento. Foram incluídos todos os casos de doença ou agravo ocupacionais notificados e encerrados nesse estado, presentes no banco de dados do Sinan, no período de 2007 a 2009.

Por meio da Portaria n ${ }^{0} 777 / 2004,{ }^{5}$ o Ministério da Saúde regulamentou a notificação compulsória de acidentes e doenças relacionados ao trabalho. Para notificação dos agravos e doenças, existem nove fichas de notificação/investigação, as quais são lançadas no Sinan. A referida portaria foi substituída pela Portaria GM/MS n ${ }^{0} 104$, de 25 de janeiro de $2011,{ }^{6}$ que, entre outros documentos oficiais, define a relação de doenças, agravos e eventos em Saúde Pública de notificação compulsória. ${ }^{5-8}$

As variáveis de estudo foram:

a) tipo de agravo relacionado ao trabalho notificado

- acidente de trabalho fatal;

- acidente de trabalho com mutilações;

- acidentes de trabalho com crianças e adolescentes;

- acidente com exposição a material biológico;

- dermatoses ocupacionais;

- intoxicações exógenas;

- lesões por esforços repetitivos (LER)/distúr- bios osteomusculares relacionadas ao trabalho (DORT);

- pneumoconioses;

- perda auditiva relacionada ao trabalho (PAIR);

- transtornos mentais relacionados ao trabalho; $\mathrm{e}$

- câncer relacionado ao trabalho;

b) município de notificação

c) características demográficas e socioeconômicas do trabalhador

- sexo (masculino/feminino/ignorado);

- idade (em anos);

- raça (parda/branca/preta/amarela/indígena/ ignorado);

- escolaridade (em anos);

- ocupação (campo aberto);

- área econômica de atuação (campo aberto);

d) situação do trabalhador no mercado de trabalho

- empregado registrado com carteira assinada ou não registrado;

- autônomo;

- servidor público;

- aposentado;

- desempregado;

- temporário;

- cooperativado;

- avulso;

- empregador;

- outra;

- ignorada/em branco; e

e) evolução do caso

- cura/cura não confirmada;

- incapacidade temporária ou permanente (parcial ou total);

- óbito por doença relacionada ao trabalho;

- óbito por outra causa;

- outra; e

- ignorada/ausente.

Para 0 cálculo da taxa de incidência, foi utilizado como numerador o número total dos agravos relacionados ao trabalho notificados por ano, e como denominador, a população economicamente ativa (PEA) no estado entre os anos de 2007 e 2009, fornecida pelo escritório estadual na Fundação Instituto Brasileiro de Geografia e Estatística (IBGE).

Os dados registrados nas fichas do Sinan foram exportados e tabulados pelo TabWin versão 3.6, vinculado ao Sinan-net, e pelo Microsoft Office Excel 2010. Para análise dos dados, foi realizada estatística 
descritiva, por meio de medidas de frequência absoluta e relativa. 0 georreferenciamento dos agravos relacionados ao trabalho, de acordo com o município de notificação, foi realizado com emprego do aplicativo ArcGis 9.1.

Este estudo foi realizado com base em dados secundários agregados, de acesso público, sem a identificação dos nomes e endereços dos sujeitos. Foi obtido o consentimento escrito do gestor estadual para a utilização dos dados do Cerest em conformidade com as Diretrizes e Normas de Pesquisa com Seres Humanos [Resolução do Conselho Nacional de Saúde (CNS) $n^{0}$ 196/1996], atualizada pela Resolução CNS $\mathrm{n}^{\circ} 466$, de 12 de dezembro de 2012.

\section{Resultados}

No Rio Grande do Norte, de 2007 a 2009, foram notificados 2.180 casos incluindo sete dos nove agravos e doenças de notificação compulsória relacionadas ao trabalho, a saber: acidentes biológicos $(\mathrm{n}=1.170 / 53,7 \%)$; acidentes graves $(\mathrm{n}=838 / 38,4 \%)$; LER/DORT $(\mathrm{n}=67 / 3,1 \%)$; intoxicações exógenas $(n=59 / 2,6 \%)$; transtornos mentais $(n=32 / 1,5 \%)$; pneumoconioses $(n=10 / 0,5 \%)$; e dermatoses $(n=4 / 0,2 \%)$. 0s agravos representados pelos acidentes foram predominantes, portanto, totalizando $92,1 \%$ dos casos notificados $(n=2.008)$. A intoxicação exógena possui uma notificação única para todos os tipos de exposição (ocupacional ou não), impossibilitando que as variáveis sejam desagregadas das diversas origens externas, motivo porque não foi possível construir uma representação tabular ou gráfica exata para esse agravo. Assim, dos 275 casos notificados, conseguiu-se identificar que 59 foram ocupacionais. Não foram notificados casos de câncer relacionado ao trabalho e de perda auditiva relacionada ao trabalho - PAIR.

Dos 167 municípios do estado do Rio Grande do Norte, $40,1 \%(n=67)$ tiveram notificações relacionadas a doenças e agravos ocupacionais. A distribuição geográfica dos casos é apresentada na Figura 1. Os municípios com maior notificação foram Natal, Mossoró e Caicó.

Evidencia-se aumento progressivo na incidência dos agravos relacionados ao trabalho notificados no Rio Grande do Norte, de 275 casos por 1000 trabalhadores em 2007 para 857,5/1000 trabalhadores em 2009 (Figura 2).
Os homens predominaram entre os acidentados e os portadores de doenças relacionadas ao trabalho. As mulheres predominaram entre os trabalhadores acometidos por acidentes envolvendo materiais biológicos. Houve um predomínio de trabalhadores na faixa etária dos 15 aos 44 anos, em todos os anos e em todos os agravos e doenças. Ademais, predominaram trabalhadores com menor escolaridade $(25,9 \%)$, embora tenha-se observado um elevado número de dados ignorados ou ausentes quanto a essa variável $(66,0 \%)$ (Tabela 1).

Em relação à ocupação, entre os profissionais de enfermagem, foram predominantes os acidentes com material biológico (99,8\%). Em trabalhadores da construção civil $(97,2 \%)$ e do setor agropecuário $(100,0 \%)$, os acidentes considerados graves foram mais frequentes (Tabela 1). No caso das doenças ocupacionais, os trabalhadores da área de serviços manuais - costureiras $(93,3 \%)$ e operadores de caixa (75\%) - foram mais acometidos por LER/DORT; os profissionais do transporte público (100\%) e do setor de segurança (100\%), por transtornos mentais; e os garimpeiros e mineiros (100\%), por pneumoconioses. Metade dos casos de dermatose ocorreu em trabalhadores da construção civil (Tabela 1).

A incapacidade temporária foi o desfecho de maior ocorrência $(n=687 / 32,4 \%)$ entre os casos investigados. Houve ainda um expressivo número de casos que tiveram o desfecho ignorado ou desconhecido: $49,8 \%$ das notificações $(\mathrm{n}=1.057)$, com predominância dos acidentes biológicos $(\mathrm{n}=1.021)$ (Tabela 2$)$.

A maioria dos acidentes graves evoluiu para incapacidade temporária $(71,9 \%), 13,0 \%$ para incapacidade parcial permanente, $0,6 \%$ para incapacidade total permanente, e 4,9\% para óbito (Tabela 2).

A maior frequência de agravos relacionados ao trabalho notificados foi observada entre empregados registrados $(\mathrm{n}=553)$. Houve ainda um grande número de registros nos quais a situação dos trabalhadores era ignorada ou desconhecida $(n=1.250)$, particularmente entre aqueles que sofreram acidentes biológicos $(n=734)$ e graves $(n=367)$ (Tabela 3$)$.

\section{Discussão}

Entre os agravos relacionados a trabalho de notificação compulsória no estado do Rio Grande do Norte, no período investigado, predominaram os acidentes 


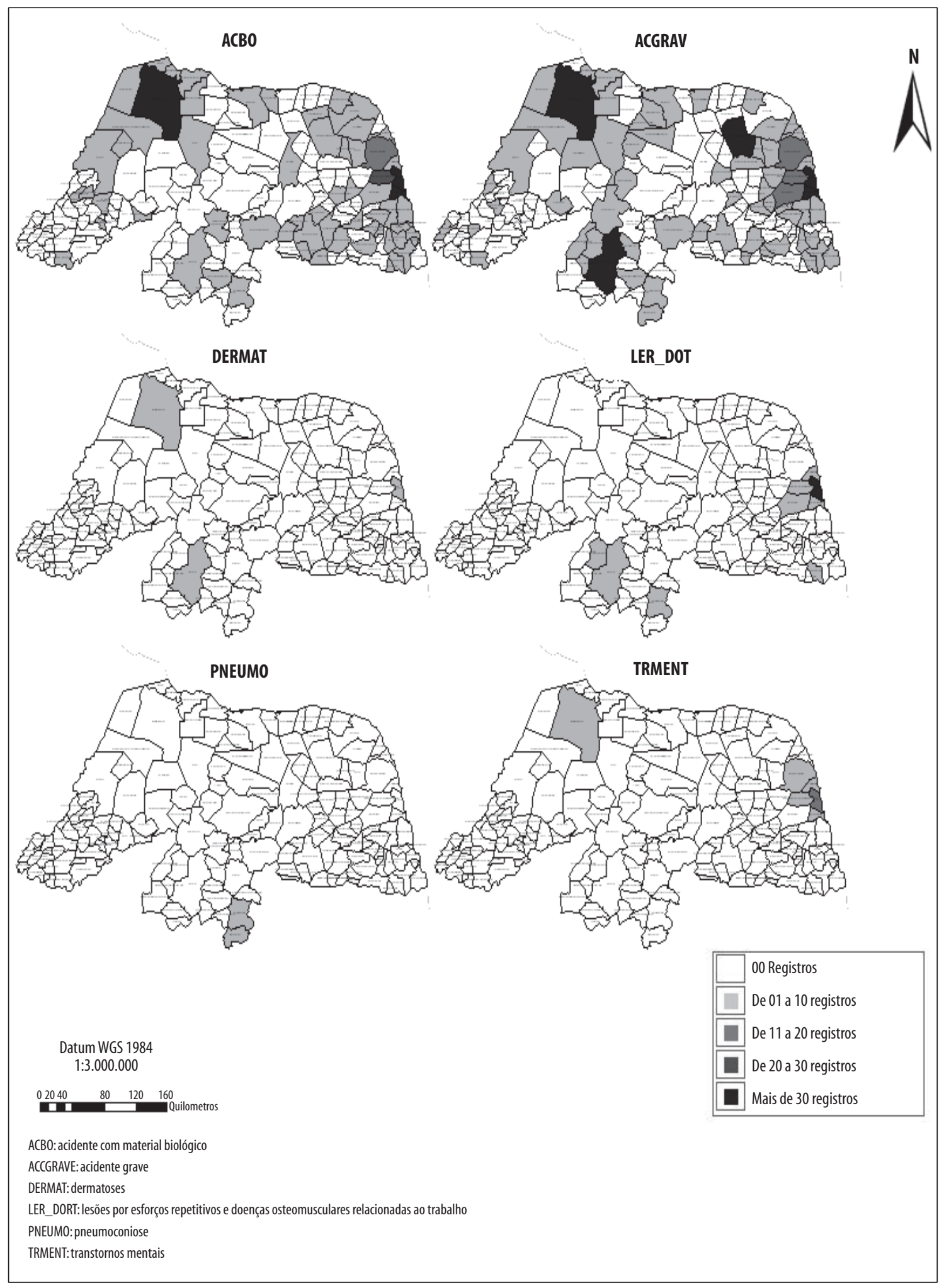

Figura 1 - Distribuição dos agravos relacionados ao trabalho notificados, segundo município de notificação, no Rio Grande do Norte, 2007 a 2009 


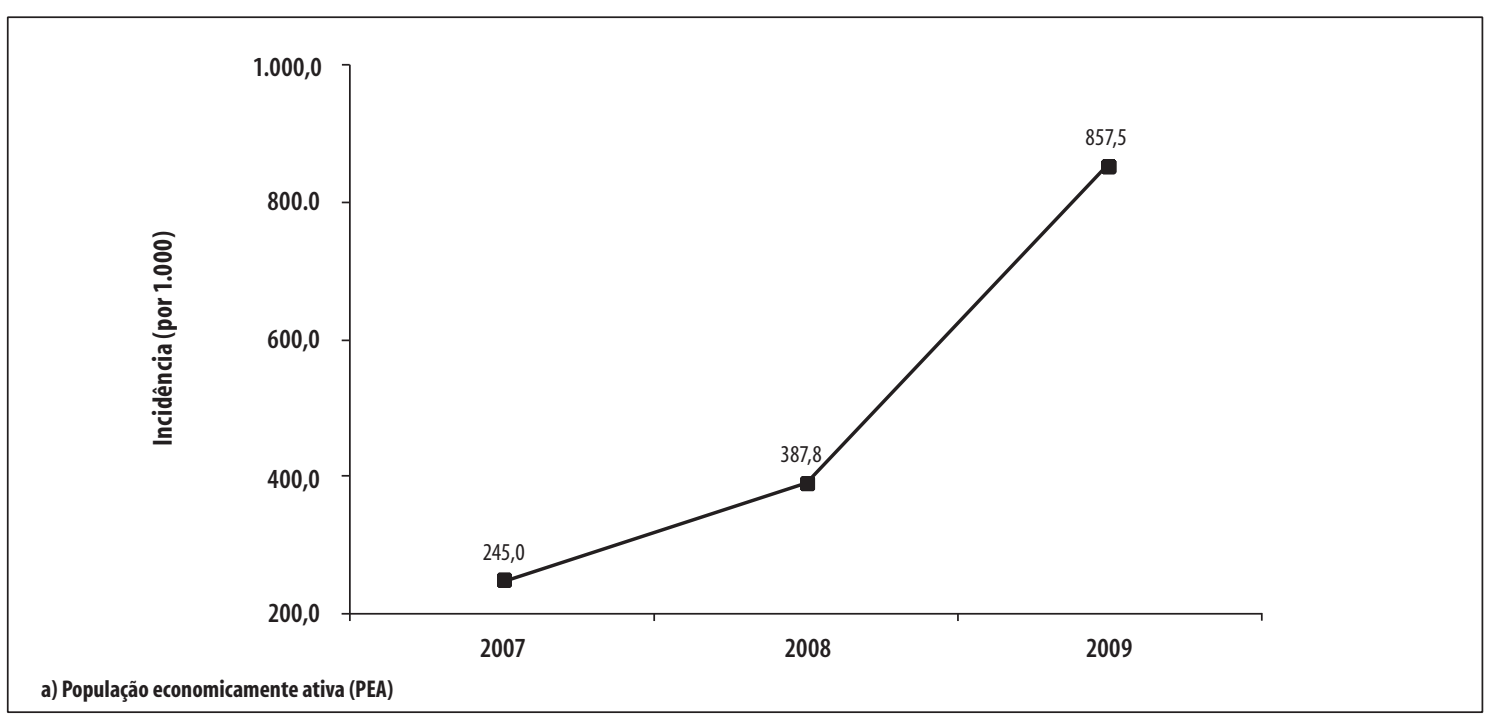

Figura 2 - Incidência (por 1000 trabalhadores $^{\mathrm{a}}$ ) dos agravos relacionados ao trabalho notificados, por ano de notificação, no Rio Grande do Norte, 2007 a 2009

Tabela 1 - Distribuição dos agravos relacionados ao trabalho segundo variáveis sociodemográficas dos trabalhadores no Rio Grande do Norte, 2007 a 2009

\begin{tabular}{|c|c|c|c|c|c|c|c|c|}
\hline \multirow{2}{*}{ Variáveis } & \multirow{2}{*}{$\begin{array}{l}\text { Acidente } \\
\text { biológico }\end{array}$} & \multirow{2}{*}{$\begin{array}{c}\text { Acidente } \\
\text { qrave }\end{array}$} & \multirow{2}{*}{ LER/DORT a } & \multirow{2}{*}{$\begin{array}{c}\text { Transtorno } \\
\text { mental }\end{array}$} & \multirow{2}{*}{ Pneumoconiose } & \multirow{2}{*}{ Dermatose } & \multicolumn{2}{|c|}{ Total } \\
\hline & & & & & & & $\mathbf{n}$ & $\%$ \\
\hline \multicolumn{9}{|c|}{ Niviogice giare } \\
\hline Masculino & 305 & 782 & 17 & 22 & 10 & 3 & 1.139 & 53,7 \\
\hline Feminino & 865 & 55 & 50 & 10 & - & 1 & 981 & 46,3 \\
\hline Ignorado/ausente & - & - & - & - & - & - & 1 & 0,0 \\
\hline \multicolumn{9}{|l|}{ Faixa etária (em anos) } \\
\hline $5-14$ & 7 & 6 & - & - & - & - & 13 & 0,6 \\
\hline $15-24$ & 208 & 184 & 3 & 3 & - & 2 & 405 & 19,1 \\
\hline $25-44$ & 760 & 482 & 49 & 20 & 5 & 2 & 1.318 & 62,1 \\
\hline $45-64$ & 182 & 140 & 13 & 8 & & - & 348 & 16,4 \\
\hline 65 ou mais & 6 & 6 & - & - & - & - & 12 & 0,6 \\
\hline Inconsistente & 7 & 15 & 2 & 1 & - & - & 25 & 1,2 \\
\hline \multicolumn{9}{|l|}{ Escolaridade (em nível de Ensino) } \\
\hline Analfabeto & 3 & 5 & - & - & 2 & 1 & 11 & 0,5 \\
\hline Fundamental incompleto & 23 & 95 & 30 & 7 & 5 & - & 160 & 7,5 \\
\hline Fundamental completo & 17 & 16 & 7 & 2 & - & 1 & 43 & 2,0 \\
\hline Médio incompleto & 15 & 36 & 1 & - & - & - & 52 & 2,5 \\
\hline Médio completo & 177 & 85 & 21 & 12 & - & - & 295 & 13,9 \\
\hline Superior incompleto & 51 & 7 & 1 & 3 & - & _- & 62 & 2,9 \\
\hline Superior completo & 63 & 9 & - & 1 & - & - & 73 & 3,4 \\
\hline Não se aplica & 7 & 15 & 2 & 1 & _- & _- & 25 & 1,2 \\
\hline Ignorada/ausente & 814 & 570 & 5 & 6 & 3 & 2 & 1.400 & 66,0 \\
\hline \multicolumn{9}{|l|}{ Ocupacão } \\
\hline Profissional de enfermagem & 567 & - & - & 1 & - & - & 568 & 26,8 \\
\hline Pedreiro/Servente de obras & - & 20 & 2 & - & - & 2 & 24 & $\begin{array}{r}20,0 \\
1,1\end{array}$ \\
\hline Agropecuário & - & 122 & - & - & - & - & 122 & 5,6 \\
\hline Costureiro & _- & 27 & 14 & 1 & _- & _- & 42 & 2,0 \\
\hline Motorista/Cobrador de ônibus & _- & - & - & 11 & - & - & 11 & 0,5 \\
\hline Soldado da Polícia Militar & - & - & - & 10 & - & - & 10 & 0,5 \\
\hline Garimpeiro & _- & _- & _- & _- & 10 & _- & 10 & 0,5 \\
\hline Operador de caixa & - & - & 3 & 1 & - & - & 4 & 0,2 \\
\hline Outras & 333 & 615 & 48 & 8 & - & 2 & 1.006 & 47,4 \\
\hline lqnorada/ausente & 270 & 54 & - & - & _- & - & 324 & 15,3 \\
\hline Total & 1.170 & 838 & 67 & 32 & 10 & 4 & 2.121 & 100,0 \\
\hline
\end{tabular}

a) LER/DORT: lesões por esforços repetitivos/doenças osteomusculares relacionadas ao trabalho 
Tabela 2 - Distribuição dos principais agravos relacionados ao trabalho segundo evolução do caso no Rio Grande do Norte, 2007 a 2009

\begin{tabular}{|c|c|c|c|c|c|c|c|}
\hline Evolução do caso & $\begin{array}{l}\text { Acidente } \\
\text { biológico }\end{array}$ & $\begin{array}{l}\text { Acidente } \\
\text { grave }\end{array}$ & $\begin{array}{l}\text { LER/ } \\
\text { DORT }^{\text {a }}\end{array}$ & $\begin{array}{c}\text { Transtorno } \\
\text { mental }\end{array}$ & Pneumoconiose & Dermatose & Tota \\
\hline Incapacidade temporária & - & 603 & 56 & 24 & 2 & 2 & 687 \\
\hline Incapacidade parcial permanente & - & 109 & 5 & 2 & 5 & - & 121 \\
\hline Alta paciente fonte negativo & 91 & - & - & - & - & - & 91 \\
\hline Alta sem conversão sorológica & 56 & - & - & - & - & - & 56 \\
\hline Cura & - & 40 & 1 & 1 & - & 1 & 43 \\
\hline Óbito pelo acidente & - & 41 & - & - & - & - & 41 \\
\hline Incapacidade total permanente & - & 5 & - & - & 2 & - & 7 \\
\hline Cura não confirmada & - & - & 4 & 3 & - & - & 7 \\
\hline Alta com conversão sorológica & 1 & - & - & - & - & - & 1 \\
\hline óbito por outras causas & - & 1 & - & - & - & - & 1 \\
\hline Abandono & 1 & - & - & - & - & - & 1 \\
\hline Outra & - & 6 & 1 & - & - & 1 & 8 \\
\hline Ignorada/ausente & 1.021 & 33 & - & 2 & 1 & - & 1.057 \\
\hline Total & 1.170 & 838 & 67 & 32 & 10 & 4 & 2.121 \\
\hline
\end{tabular}

a) LER/DORT: lesões por esforços repetitivos/doenças osteomusculares relacionadas ao trabalho

biológicos, seguidos pelos distúrbios osteomusculares, intoxicações exógenas, transtornos mentais, pneumoconioses e dermatoses. Não foram notificados casos de câncer relacionado ao trabalho e casos de PAIR. No entanto, LER/DORT, câncer e PAIR foram agravos frequentes entre trabalhadores no país, e são passíveis de agravamento com a alta exploração dos trabalhadores, flexibilização dos contratos de trabalho e importação de tecnologias sujas, decorrentes da globalização. ${ }^{3}$

Os achados desta pesquisa sugerem aumento na notificação dos agravos relacionados ao trabalho após a implantação da Portaria n ${ }^{0}$ 104/2011. ${ }^{6}$ Contudo, apesar dos avanços, estima-se que esse número seja bastante superior. Estudos prévios realizados no Brasil indicaram que a ausência de notificação não indica, necessariamente, a inexistência de casos, e sim subnotificação. ${ }^{9-13}$ 0 acidente do trabalho é registrado no protocolo da
Comunicação de Acidente de Trabalho (CAT), conforme determinação da Previdência Social, e disponibilizado no Sinan-net, por via impressa e eletrônica. Ainda assim, de acordo com a literatura, menos de $30 \%$ dos acidentes ocorridos no Brasil são notificados. ${ }^{11,14-16}$

Em sua maioria, os acidentes graves foram identificados em municípios mais desenvolvidos economicamente, no âmbito da construção civil, mecanização da agricultura e indústria - Natal, Mossoró, Caicó e João Câmara -, e nas ocupações em que há, proporcionalmente, um maior número de trabalhadores no estado, caso da construção civil e da agricultura. Além disso, são nessas cidades que se encontram as unidades das regionais de saúde da Secretaria de Estado de Saúde Pública do Rio Grande do Norte (URSAP), o que favorece a concentração e a valorização do registro das informações em saúde. 


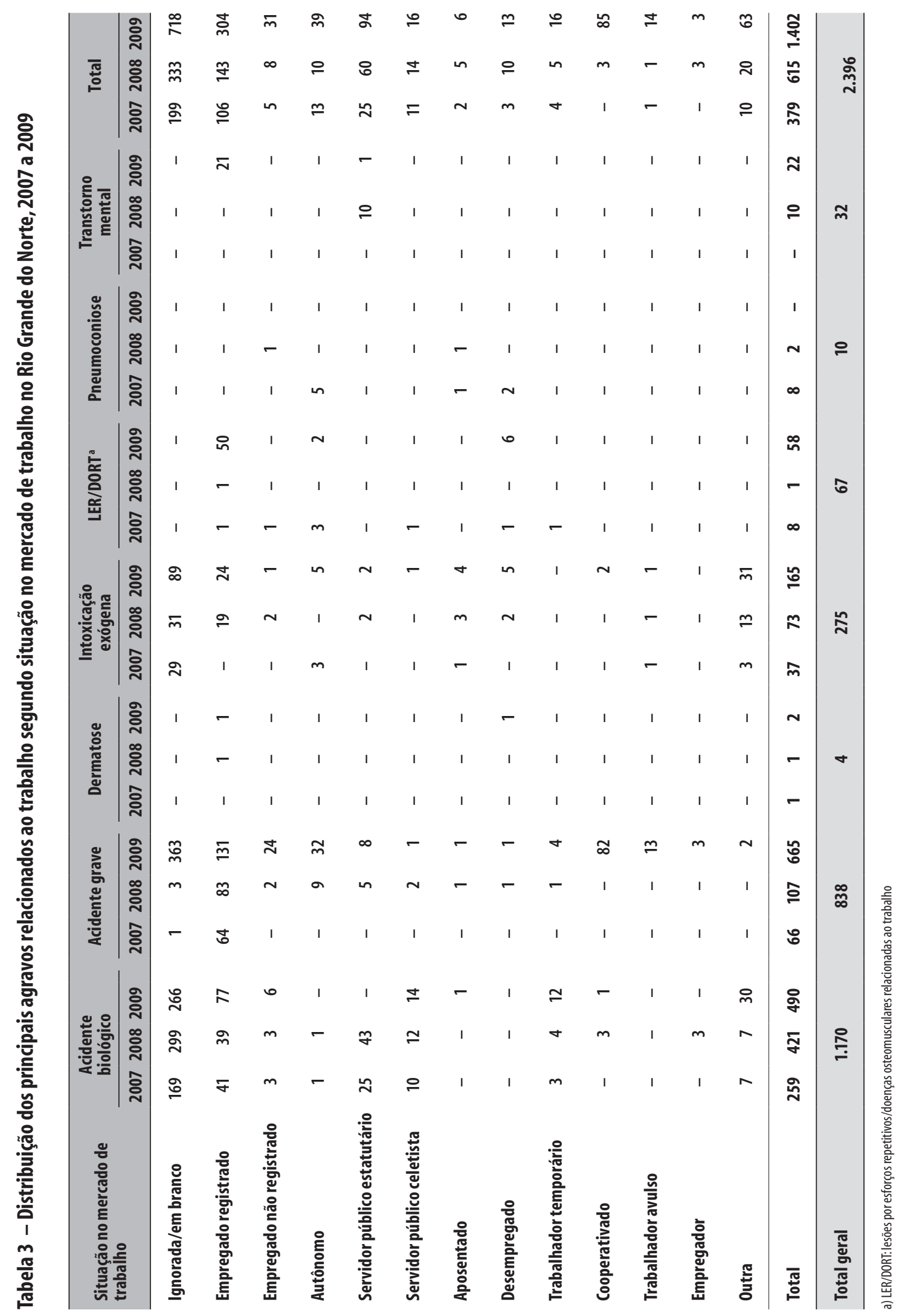


Estudos constataram que a maior parte dos acidentes atinge homens jovens e produtivos, que trabalham em indústrias, na construção civil, no setor de transportes e na agricultura, locais onde mais ocorrem acidentes graves. ${ }^{15,17,18}$ Isso reflete tanto os riscos inerentes ao exercício dessas funções quanto 0 descumprimento do uso de equipamentos e medidas de segurança.

Os acidentes biológicos ocorreram em municípios diversificados. Acredita-se que tal fato esteja relacionado à existência de profissionais e unidades de saúde em todos os municípios, o que potencializa 0 aumento de ocorrência de acidentes, bem como sua notificação. Apesar da existência dessas notificações, Silva e colaboradores ${ }^{11}$ constataram em seu estudo que, todavia, é expressiva a subnotificação e a falta de registros de investigações dos casos, além da ausência de acompanhamento do desfecho.

0 elevado número de casos de acidentes biológicos com desfecho ignorado ou desconhecido demonstra, além da incompletude dos dados, perda de acompanhamento do profissional e falta de registro de soroconversão pós-exposição biológica, situação evidenciada também no estudo de Silva e cols. ${ }^{11}$

Houve um predomínio no número de casos de acidentes biológicos entre os profissionais de enfermagem, categoria historicamente mais acometida por esse tipo de acidente, justamente pelas especificidades de um processo de trabalho de cuidado ininterrupto $\mathrm{e}$ contínuo de parte desses profissionais, portanto sob maior exposição. $14,17,19-21$

Os transtornos mentais apresentaram casos apenas nos municípios de Natal e Mossoró, cidades com maior densidade demográfica e concentradoras das atividades produtivas consideradas mais estressantes e perigosas, regimes de turnos alternados e trabalho noturno - como os de profissionais dos setores de segurança e dos transportes públicos. ${ }^{22,23}$

A pneumoconiose acometeu principalmente os garimpeiros e mineiros da região central potiguar, estando, portanto, relacionada às atividades de mineração desenvolvidas nessa região. Porém, foram registrados casos da doença em apenas dois municípios: Equador e Parelhas. Essa situação é ocasionada, geralmente, pela dificuldade de estabelecer o nexo causal da pneumoconiose, que apresenta longos períodos de latência e permanece irreconhecível até que seus sintomas clínicos se manifestem. ${ }^{3}$
Os casos de LER/DORT foram notificados na região do Seridó, com concentração em Caicó, e na região da Grande Natal, em trabalhadores da área de serviços manuais como costureiros e operadores de caixa. Pode-se inferir que a concentração de casos notificados em Caicó ocorra devido à presença da sede da IV Regional de Saúde do Estado, como também pela grande quantidade de bordadeiras, fabricantes de queijos artesanais e bonelarias no município. $\mathrm{Na}$ Grande Natal, a maior presença de indústrias nessa área explica a concentração de casos de LER/DORT, relacionados à execução de atividades com movimentos repetitivos. ${ }^{24-25}$

Quanto ao acompanhamento da evolução nos casos de LER/DORT e transtornos mentais, observou-se incapacidade temporária $(83,6 \%$ e 75,0\% respectivamente) e parcial permanente $(7,5 \%$ e $6,2 \%$ respectivamente), além de cura não confirmada ( $5,9 \%$ e 9, $4 \%$ respectivamente). Esses agravos impõem limitações físicas e emocionais ao trabalhador, muitas vezes comprometendo seu retorno ao trabalho, e necessitam, para seu tratamento, de um longo período de acompanhamento multiprofissional., ${ }^{3,1426}$ Destacam-se ainda os acidentes graves, que causam mais incapacidades e afastamentos. Estudos demonstram o impacto socioeconômico desses afastamentos para a empresa, o trabalhador, a Previdência Social, e para toda a sociedade. ${ }^{3,12,17,22,27-29}$

Em relação ao sexo, observou-se que em 2009, os homens foram os mais acometidos pelos agravos relacionados ao trabalho no Rio Grande do Norte. Em 2007 e 2008, ao contrário, foi o sexo feminino o mais atingido. Essa realidade reflete o predomínio de homens em atividades de maior risco, como construção civil e mineração. Por outro lado, as mulheres foram as mais vitimadas pelos acidentes biológicos: historicamente, há um predomínio da força de trabalho feminina na área da Saúde, principalmente na Enfermagem. . $^{10,12,15,24,17}$

No que se refere à faixa etária, houve um maior número de casos em trabalhadores de 15 a 44 anos, em todo o período analisado, o que está em concordância com o fato de ser maior a exposição desses indivíduos enquanto componentes da população economicamente ativa.

Os eventos ocorreram com mais frequência entre os trabalhadores de menor nível educacional. Outros estudos também evidenciaram que os trabalhadores 
com baixa qualificação profissional, menores salários e baixo poder decisório são os que mais se acidentam e adoecem. ${ }^{11,12,17,27}$

Observou-se uma concentração de trabalhadores no setor formal da economia. Este achado tem semelhança com outros estudos e evidencia que as estatísticas oficiais dos agravos relacionados ao trabalho no Brasil, tradicionalmente, têm origem em dados da CAT, instrumento de informação do Instituto Nacional do Seguro Social (INSS) que gera benefícios previdenciários aos trabalhadores do mercado formal. ${ }^{17,20,23,24}$

0 presente estudo evidenciou que os sistemas de informações em saúde do país continuam a demandar melhores registros, tanto de cobertura como de qualidade dos dados. 0 número considerável de dados em branco ou ignorados, encontrados em todas as variáveis consideradas, reforça essa questão.

Portanto, é preciso ter em conta, como limitação do estudo, o uso dos dados produzidos pelo sistema de vigilância. Ainda que o Sinan seja a melhor fonte para análise de dados epidemiológicos, sabe-se que problemas do sistema impossibilitam um diagnóstico situacional da Vigilância em Saúde do Trabalhador sobre bases mais profundas e acuradas., ${ }^{3,10,12,30}$

\section{Referências}

1. Aragón A, Partanen T, Felknor S, Corriols M. Social determinants of workers' health in Central America. Int J Occup Environ Health. 2011 JulSep;17(3):230-7.

2. Conselho Nacional de Secretários de Saúde (BR). Vigilância em saúde: parte 1. Brasília: CONASS, 2011. Capítulo 7, Vigilância em saúde do trabalhador; p. 232-60.

3. International Labour Organization. The prevention of occupational diseases. Geneva: International Labour Organization; 2013.

4. Ministério do Trabalho e Emprego (BR). Política nacional de segurança e saúde do trabalhador: versão de 12/11/2004. Brasília: Ministério do Trabalho e Emprego; 2004.

5. Brasil. Ministério da Saúde. Portaria GM/MS $\mathrm{n}^{\circ} 777$, de 28 de abril de 2004. Dispõe sobre os procedimentos técnicos para a notificação compulsória de agravos à saúde do trabalhador em rede de serviços sentinela específica, no Sistema
A inclusão dos agravos relacionados ao trabalho na lista de notificação compulsória, desde 2004, tem permitido o desvelamento da realidade desses agravos no país, subsidiando, com maior fidedignidade, as políticas públicas de saúde e segurança para o trabalhador.

Os resultados deste estudo representam o primeiro passo de uma importante tarefa de avaliação epidemiológica da situação do trabalhador no estado do Rio Grande do Norte, no caminho do aprimoramento do sistema de informação, assim como das ações de promoção, prevenção, controle e reabilitação da saúde do trabalhador.

\section{Contribuição dos autores}

Cavalcante CAA, Cavalcante EFO, Santos RS, Martins RL, Silva ET e Silveira EA contribuíram na concepção e delineamento do estudo, análise e interpretação dos resultados, redação e revisão crítica do conteúdo intelectual e aprovação da versão final do manuscrito.

Todos os autores aprovaram a versão final do manuscrito e declaram serem responsáveis por todos os aspectos do trabalho, garantindo sua precisão e integridade.

Único de Saúde - SUS. Diário Oficial da República Federativa do Brasil, Brasília (DF), 2004 abr 29; Seção 1:37.

6. Brasil. Ministério da Saúde. Portaria GM/MS n 104 , de 25 de janeiro de 2011. Define as terminologias adotadas em legislação nacional, conforme o disposto no Regulamento Sanitário Internacional 2005 (RSI 2005), a relação de doenças, agravos e eventos em saúde pública de notificação compulsória em todo o território nacional e estabelece fluxo, critérios, responsabilidades e atribuições aos profissionais e serviços de saúde. Diário Oficial da República Federativa do Brasil, Brasília (DF), 2011 jan 26; Seção 1:37.

7. Brasil. Ministério da Saúde. Portaria GM/MS 2472, de 31 de agosto de 2010. Define a relação de doenças, agravos e eventos em saúde pública de notificação compulsória e estabelece fluxo, critérios, responsabilidades e atribuições aos profissionais e serviços de saúde. Diário Oficial da República Federativa do Brasil, Brasília (DF), 2010 set 1; Seção 1:50. 
8. Ministério da Saúde (BR). Informações em saúde [Internet]. 2010 [citado 2010 out 1]. Disponível em: http://portal.saude.gov.br/portal/arquivos/pdf/ vivapres $7 . p d f$

9. Facchini LA, Nobre LCC, Faria NMX, Fassa AG, Thumé E, Tomasi E, et al. Sistema de informação em saúde do trabalhador: desafios e perspectivas para o SUS. Cienc Saude Coletiva. 2005 out-dez;10(4):857-67.

10. Scherer V, Miranda F, Sarquis L, Lacerda M. Sinan Net: um sistema de informação à vigilância na saúde do trabalhador. Cogitare Enferm. 2007 julset;12(3):330-7.

11. Binder MCP, Cordeiro R. Sub-registro de acidentes do trabalho em localidade do Estado de São Paulo, 1997. Rev Saude Publica. 2003 ago;37(4):409-16.

12. Silva AID, Machado JMH, Santos EGOB, Marziale MHP. Acidentes com material biológico relacionados ao trabalho: análise de uma abordagem institucional. Rev Bras Saude Ocup. 2011 jul-dez;36(124):265-73.

13. Bortoleto MSS, Nunes EFPA, Haddad MCL, Reis GAX. Acidentes de trabalho em um pronto atendimento do sistema único de saúde. Espaç Saude. 2011 dez;13(1):91-7.

14. Scussiato LA, Céspedes LDM, Sarquis LMM, Stein Junior AV, Miranda FMA. A análise dos agravos relacionados ao trabalho notificados pela unidade saúde do trabalhador. Rev Min Enferm. 2010 janmar;14(1):88-95.

15. Santos SS, Costa NA, Mascarenhas MDM. Caracterização das exposições ocupacionais a material biológico entre trabalhadores de hospitais no Município de Teresina, Estado do Piauí, Brasil, 2007 a 2011. Epidemiol Serv Saude. 2013 janmar;22(1):165-70

16. García AM, Gadea R. Estimaciones de incidencia y prevalencia de enfermedades de origen laboral em España. Aten Primaria. 2008 sep;40(9):439-46.

17. García Gomez M, Castañeda Lopez RC. Desigualdades interterritoriales em lacompensacion de las enfermedades profesionales em España de 1990 a 2007. Gac Sanit. 2009 sep-oct;23(5):373-9.

18. Vilela LVO, Assunção AA. Os mecanismos de controle da atividade no setor de teleatendimento e as queixas de cansaço e esgotamento dos trabalhadores. Cad Saude Publica. 2004 jul-ago;20(4):1069-78.
19. Rios MA, Nery AA, Alves MS, Jesus CS. Acidentes e doenças relacionadas ao trabalho em Jequié, Bahia, registrados no Instituto Nacional de Seguridade Social, 2008-2009. Epidemiol Serv Saude. 2012 jun;21(2):315-24.

20. Souza NSS, Santana VS. Incidência de doenças musculoesqueléticas incapacitantes. Cad Saude Publica. 2011 nov;27(11):2124-34.

21. Sarquis LMM, Felli VE, Miranda FMA, Guimarães HV, Oliveira GP. A adesão ao protocolo de monitoramento dos trabalhadores de saúde após exposição a fluidos biológicos: uma problemática vivenciada em um ambulatório de saúde do trabalhador no Paraná. Cogitare Enferm. 2005 maiago;10(2):47-53.

22. Miranda FMD, Scussiato LA, Kirchhof ALC, Cruz EDA, Sarquis LMM. Caracterização das vítimas e dos acidentes de trabalho fatais. Rev Gaucha Enferm. 2012 jun;33(2):45-51.

23. Santana VS, Xavier C, Moura MCP, Oliveira R, EspiritoSanto JS, Araújo G. Gravidade dos acidentes de trabalho atendidos em serviços de emergência. Rev Saude Publica. 2009 out;43(5):750-60.

24. Hennington EA, Monteiro M. O perfil epidemiológico dos acidentes de trabalho no Vale dos Sinos e o sistema de vigilância em saúde do trabalhador. Hist Cienc Saude-Manguinhos. 2006 outdez;13(4):865-76.

25. Vecchio N, Scuffham PA, Hilton MF, Whiteford HA. Work-related injury in the nursing profession: an investigation of modifiable factors. J Adv Nurs. 2011 May;67(5):1067-78.

26. Alves MMM, Nomellini PF, Pranchevicius MCS. Mortalidade por acidente de trabalho no Estado do Tocantins, Brasil: estudo descritivo, 2000-2010. Epidemiol Serv Saude. 2013 jun;22(2):243-54.

27. Franco T, Druck G, Seligmann-Silva E. As novas relações de trabalho, o desgaste mental do trabalhador e os transtornos mentais no trabalho precarizado. Rev Bras Saude Ocup. 2010 juldez;35(122):229-48.

28. Galdino A, Santana VS, Ferrite S. Os centros de referência em saúde do trabalhador e a notificação de acidentes de trabalho no Brasil. Cad Saude Publica. 2012 jan;28(1):145-59. 
29. Souza NSS, Santana VS, Albuquerque-Oliveira PR, Barbosa-Branco A. Doenças do trabalho e benefícios previdenciários relacionados à saúde, Bahia, 2000. Rev Saude Publica. 2008 ago;42(4):630-8.
30. Cardoso EM. Morbimortalidade relacionada ao trabalho no Amazonas. Epidemiol Serv Saude. 2014 mar;23(1):143-53.

Recebido em 24/05/2014 Aprovado em 10/10/2014 